\title{
Evaluation of Blood Pressure and Heart Rate in Patients with Hypertension Who Received Tapentadol Extended Release for Chronic Pain: A Post Hoc, Pooled Data Analysis
}

\author{
David M. Biondi $\cdot$ Jim Xiang $\cdot$ Mila Etropolski • \\ Bruce Moskovitz
}

Published online: 11 June 2014

(C) The Author(s) 2014. This article is published with open access at Springerlink.com

\begin{abstract}
Background and Objectives Hypertension is one of the most common co-existing conditions in patients with chronic pain, and the potential effects of an analgesic on heart rate and blood pressure are of particular concern for patients with hypertension. The purpose of this analysis was to evaluate changes in blood pressure and heart rate with tapentadol extended release (ER) treatment in patients with hypertension.

Methods We performed a post hoc analysis of data pooled from three randomized, placebo- and active-controlled, phase III studies of tapentadol ER for managing chronic osteoarthritis knee (NCT00421928, NCT00486811) or low back (NCT00449176) pain (15-week, double-blind treatment period). Data were independently analyzed for patients with a listed medical history of hypertension at baseline and patients with at least one listed concomitant antihypertensive medication at baseline. Heart rate, systolic blood pressure (SBP), and diastolic blood pressure (DBP) were measured at each visit.

Results In patients with a listed medical history of hypertension $(n=1,464)$, least-squares mean (LSM [standard error (SE)]) changes from baseline to endpoint with placebo, tapentadol $\mathrm{ER}$, and oxycodone $\mathrm{HCl}$
\end{abstract}

ClinicalTrial.gov Identifiers: NCT00421928, NCT00486811, and NCT00449176.

D. M. Biondi $(\varangle) \cdot$ B. Moskovitz

US Medical Affairs, Janssen Scientific Affairs, LLC, 1125 Trenton Harbourton Road, Titusville, NJ 08560, USA e-mail: dbiondi@its.jnj.com

J. Xiang · M. Etropolski

Janssen Research \& Development, LLC, 1125 Trenton

Harbourton Road, Titusville, NJ 08560, USA controlled release (CR), respectively, were $-0.7(0.44), 0.2$ $(0.43)$, and $-0.9(0.45)$ beats per minute $(\mathrm{bpm})$ for heart rate; $-2.4(0.64),-2.7(0.64)$, and $-3.7(0.67) \mathrm{mmHg}$ for SBP; and $-1.0(0.39),-1.3(0.39)$, and $-2.3(0.41) \mathrm{mmHg}$ for DBP; in patients with at least one listed concomitant antihypertensive medication $(n=1,376)$, the LSM (SE) changes from baseline to endpoint were $-0.6(0.45), 0.1$ (0.44), and $-0.7(0.47) \mathrm{bpm}$ for heart rate; $-1.8(0.66)$, $-3.3(0.65)$, and $-3.7(0.69) \mathrm{mmHg}$ for SBP; and -0.7 (0.40), $-1.4(0.40)$, and $-2.3(0.42) \mathrm{mmHg}$ for DBP.

Conclusion No clinically meaningful mean changes in heart rate or blood pressure were observed for the evaluated cohorts of patients with hypertension who were treated with tapentadol ER (100-250 mg twice daily).

\section{Key Points}

Mean heart rate, systolic blood pressure, and diastolic blood pressure values remained generally unchanged over 15 weeks of treatment with placebo, tapentadol extended release (ER; 100-250 mg twice daily), or oxycodone $\mathrm{HCl}$ controlled release (20-50 mg twice daily) in two patient cohorts: patients with a listed medical history of hypertension at baseline and patients with at least one listed concomitant antihypertensive medication at baseline

Findings suggest that tapentadol ER is a reasonable option when considering treatments for the management of chronic pain in patients with hypertension, although a personalized assessment of patient risk and prudent clinical judgment are recommended 


\section{Introduction}

Hypertension is one of the most common co-existing medical conditions in patients with chronic pain [1, 2]. In a study of 29,132 patients with osteoarthritis [1], hypertension was the most common co-existing condition $(52 \%$ of patients). Acetaminophen (paracetamol), NSAIDs, and cyclo-oxygenase (COX)-2 inhibitors are often recommended for patients initiating pharmacological therapy for the management of osteoarthritis [3-5] and low back pain $[3,6,7]$; however, use of acetaminophen [8], NSAIDs [9], and COX-2 inhibitors [10] has been associated with increases in blood pressure, which may be particularly problematic for patients with pre-existing hypertension [3, $8,9]$.

Serotonin norepinephrine reuptake inhibitors (SNRIs) are often prescribed for the management of chronic musculoskeletal pain, including chronic osteoarthritis pain and chronic low back pain [11]. SNRIs have been associated with increases in blood pressure and heart rate [12], possibly related to the norepinephrine reuptake inhibitor component of their pharmacologic activity [13]. An extended-release (ER) formulation of the centrally acting analgesic tapentadol, which has both $\mu$-opioid receptor agonist and norepinephrine reuptake inhibitor activities [14-16], has demonstrated efficacy and safety for the management of moderate to severe, chronic pain in adults when a continuous, around-the-clock opioid analgesic was needed for an extended period of time [17]. Phase III clinical trials of tapentadol ER included studies in patients with moderate to severe, chronic osteoarthritis hip or knee pain $[18,19]$, low back pain $[19,20]$, and pain related to diabetic peripheral neuropathy [21].

A pooled analysis [22] of data from three randomized, placebo- and active-controlled, phase III studies with 15 weeks of double-blind treatment (two studies in chronic osteoarthritis knee pain [18], one study in chronic low back pain [20]) demonstrated that the efficacy of tapentadol ER (100-250 mg twice daily) was non-inferior to that of oxycodone $\mathrm{HCl}$ controlled release (CR; 20-50 mg twice daily) for the management of moderate to severe, chronic pain. In that analysis [22], the incidences of constipation, nausea, vomiting, and the composite of nausea and/or vomiting were significantly lower with tapentadol ER than with oxycodone CR. An analysis of data pooled from the same three studies demonstrated that tapentadol ER (100-250 mg twice daily) treatment $(n=920)$ was not associated with clinically meaningful changes in mean vital sign measures, including heart rate and blood pressure [23]. The current post hoc pooled data analysis used data from those three studies to evaluate changes in blood pressure and heart rate in patients with a listed medical history of hypertension or at least one listed concomitant antihypertensive medication at baseline who were treated with tapentadol ER.

\section{Methods}

\subsection{Patients}

Data were pooled from patients with a listed medical history of hypertension or at least one listed concomitant antihypertensive medication at baseline who had participated in one of three double-blind, placebo- and activecontrolled, phase III studies of tapentadol ER for moderate to severe, chronic osteoarthritis knee pain (ClinicalTrials.gov Identifiers: NCT00421928 [18] or NCT00486811) or low back pain (NCT00449176 [20]). Similar eligibility criteria were used in all three studies; these criteria have been described previously [22]. Patients with uncontrolled hypertension were not permitted to participate in any of the three studies.

Patients were not permitted to use antipsychotics, anticonvulsants, antiparkinsonian medications, tricyclic antidepressants, or SNRIs within 14 days prior to screening or during the study because the possible analgesic effects of these medications could confound primary assessments of analgesic efficacy. The use of monoamine oxidase inhibitors was prohibited within 14 days prior to screening and throughout the study. Systemic corticosteroids were not permitted during the study or within 4 weeks of screening for orally administered corticosteroids, 8 weeks for soft tissue- or intramuscularly administered corticosteroids, 3 months for intra-articularly administered corticosteroids, and 6 months for depot-injected corticosteroids.

\subsection{Study Design}

The three studies were conducted in accordance with the ethical principles of the Declaration of Helsinki and Good Clinical Practice. The protocols were reviewed and approved by institutional review boards, and patients provided informed consent. The three studies included in this analysis had similar designs, as outlined previously [22] and briefly summarized here. Each randomized, doubleblind, multicenter, placebo- and active-controlled, phase III study included a 15-week, double-blind treatment period (3 weeks titration, 12 weeks maintenance). Patients were randomized (1:1:1) to receive placebo, tapentadol ER, or oxycodone CR. For the first 3 days of titration, patients received twice-daily doses of placebo, tapentadol ER $50 \mathrm{mg}$, or oxycodone $\mathrm{HCl} \mathrm{CR} 10 \mathrm{mg}$; active treatment doses were then increased for the next 4 days to tapentadol 
ER $100 \mathrm{mg}$ twice daily or oxycodone $\mathrm{HCl}$ CR $20 \mathrm{mg}$ twice daily (minimum therapeutic doses permitted to remain eligible for treatment during the maintenance period in these studies). Patients were then individually titrated to the dose that provided the most favorable balance between pain reduction and tolerability for the remaining 2 weeks of titration. Under physician supervision, patients could increase or decrease their doses of study medication by $50 \mathrm{mg}$ twice daily for tapentadol ER or $10 \mathrm{mg}$ twice daily for oxycodone $\mathrm{HCl} \mathrm{CR}$ within the prespecified therapeutic ranges (tapentadol ER 100-250 mg twice daily; oxycodone $\mathrm{HCl}$ CR 20-50 mg twice daily); doses could be increased at a minimum of 3-day intervals or decreased with no time restriction. Acetaminophen $(\leq 1,000 \mathrm{mg} /$ day $)$ was permitted during titration except for the last 3 days. Although patients made an effort to maintain steady dose concentrations of study medication throughout the maintenance period, dose adjustments within the prespecified therapeutic ranges were permitted under physician supervision to maintain the best possible balance of efficacy and tolerability.

\subsection{Study Evaluations and Statistical Analyses}

Vital signs, including heart rate and supine or sitting blood pressure, were recorded after patients had rested for $5 \mathrm{~min}$ at each study visit in each study and were analyzed for all randomized patients who had received one or more dose of their study medication (safety population). For this post hoc data analysis, heart rate, systolic blood pressure (SBP), and diastolic blood pressure (DBP) were independently analyzed for the following two cohorts: patients with a listed medical history of hypertension at baseline and patients with at least one listed concomitant antihypertensive medication at baseline.

These analyses were performed using SAS ${ }^{\circledR}$ software version 9.1 (SAS Institute, Cary, NC, USA). Descriptive statistics were used to summarize absolute values and changes from baseline to endpoint for heart rate and blood pressure by treatment group. The baseline value was defined as the last measured heart rate or blood pressure value prior to the first dose of randomized treatment, and the endpoint value was defined as the last measured, postbaseline heart rate or blood pressure value during doubleblind treatment. Between-group differences in the leastsquares mean (LSM) change from baseline in heart rate and blood pressure were evaluated using an analysis of covariance (ANCOVA) model, with treatment, sex, baseline body mass index group ( $<30$ or $\geq 30 \mathrm{~kg} / \mathrm{m}^{2}$ ), and study as factors, and with age and baseline value as covariates.

The percentage of patients with changes from baseline in heart rate in each of the following categories was evaluated by treatment group for each cohort: $<0$ beats per minute (bpm), $\geq 0$ to $<10 \mathrm{bpm}, \geq 10$ to $<15 \mathrm{bpm}, \geq 15$ to $<20 \mathrm{bpm}$, and $\geq 20 \mathrm{bpm}$. Similar evaluations were performed to determine the percentages of patients with changes from baseline in SBP or DBP of $<0 \mathrm{mmHg}, \geq 0$ to $<5 \mathrm{mmHg}, \geq 5$ to $<10 \mathrm{mmHg}, \geq 10$ to $<15 \mathrm{mmHg}$, and $\geq 15 \mathrm{mmHg}$. The overall distributions of patients in the categories of changes from baseline in heart rate, SBP, and DBP were compared between the tapentadol ER and placebo groups, between the oxycodone $\mathrm{CR}$ and placebo groups, and between the tapentadol ER and oxycodone CR groups in each of the evaluated cohorts using the row mean score option of the Cochran-Mantel-Haenszel test. The percentages of patients with sustained (observed over three or more consecutive visits) increases from baseline in SBP or DBP of $\geq 5, \geq 10$, and $\geq 15 \mathrm{mmHg}$ were also evaluated.

Treatment-emergent adverse events (TEAEs) were recorded throughout the studies. Cardiovascular TEAEs and serious cardiovascular TEAEs are summarized briefly.

\section{Results}

\subsection{Patients}

Data were analyzed for patients with a listed medical history of hypertension ( $n=1,464)$ and patients with at least one listed concomitant antihypertensive medication $(n=1,376)$. In patients with a listed medical history of hypertension, $86.9 \%(1,272 / 1,464)$ also had at least one listed concomitant antihypertensive medication. Demographic and baseline characteristics were generally comparable across treatment groups in both patient cohorts (Table 1). The most commonly listed concomitant antihypertensive medications (listed for $\geq 100$ patients) were lisinopril, ramipril, atenolol, metoprolol, and hydrochlorothiazide.

\subsection{Heart Rate}

Mean heart rate values over time are summarized in Fig. 1. Across both patient cohorts, there were no significant differences in the LSM changes from baseline to endpoint in heart rate between the tapentadol ER and placebo groups, between the oxycodone $\mathrm{CR}$ and placebo groups, or between the tapentadol ER and oxycodone CR groups. LSM changes from baseline to endpoint in heart rate were $<1 \mathrm{bpm}$ in both patient cohorts (Table 2).

The percentages of patients in each of the categories of heart rate changes $(<0, \geq 0$ to $<10, \geq 10$ to $<15, \geq 15$ to $<20$, and $\geq 20 \mathrm{bpm}$ ) from baseline to endpoint in both patient cohorts are summarized in Fig. 2. There were no significant differences in the overall distribution of categories of heart rate changes from baseline to endpoint 
Table 1 Baseline and demographic characteristics for patients with a listed medical history of hypertension and for patients with at least one listed concomitant antihypertensive medication (safety population)

\begin{tabular}{|c|c|c|c|c|c|c|}
\hline \multirow[t]{2}{*}{ Characteristic } & \multicolumn{3}{|c|}{ Listed medical history of hypertension } & \multicolumn{3}{|c|}{ Listed concomitant antihypertensive medication } \\
\hline & $\begin{array}{l}\text { Placebo } \\
(n=477)\end{array}$ & $\begin{array}{l}\text { Tapentadol ER } \\
(n=488)\end{array}$ & $\begin{array}{l}\text { Oxycodone CR } \\
(n=499)\end{array}$ & $\begin{array}{l}\text { Placebo } \\
(n=446)\end{array}$ & $\begin{array}{l}\text { Tapentadol ER } \\
(n=461)\end{array}$ & $\begin{array}{l}\text { Oxycodone CR } \\
(n=469)\end{array}$ \\
\hline \multicolumn{7}{|l|}{ Age } \\
\hline Mean (SD) [years] & $61.6(9.67)$ & $61.3(10.69)$ & $60.9(10.22)$ & $62.1(9.68)$ & $61.0(10.89)$ & $61.2(10.35)$ \\
\hline \multicolumn{7}{|l|}{ Category $[n(\%)]^{\mathrm{a}}$} \\
\hline$<65$ years & 294 (61.6) & $296(60.7)$ & $314(62.9)$ & $264(59.2)$ & $286(62.0)$ & $288(61.4)$ \\
\hline$\geq 65$ to $<75$ years & $141(29.6)$ & $136(27.9)$ & $131(26.3)$ & $139(31.2)$ & $127(27.5)$ & $129(27.5)$ \\
\hline$\geq 75$ years & $42(8.8)$ & $56(11.5)$ & $54(10.8)$ & $43(9.6)$ & $48(10.4)$ & $52(11.1)$ \\
\hline \multicolumn{7}{|l|}{$\operatorname{Sex}[n(\%)]$} \\
\hline Male & $162(34.0)$ & $158(32.4)$ & $189(37.9)$ & $161(36.1)$ & 155 (33.6) & $178(38.0)$ \\
\hline Female & $315(66.0)$ & $330(67.6)$ & $310(62.1)$ & $285(63.9)$ & $306(66.4)$ & $291(62.0)$ \\
\hline \multicolumn{7}{|l|}{$\operatorname{Race}[n(\%)]^{\mathrm{a}}$} \\
\hline White & $402(84.3)$ & $396(81.1)$ & $410(82.2)$ & $375(84.1)$ & $378(82.0)$ & $391(83.4)$ \\
\hline Black & $51(10.7)$ & $68(13.9)$ & $54(10.8)$ & $45(10.1)$ & $62(13.4)$ & $45(9.6)$ \\
\hline Hispanic & $12(2.5)$ & $15(3.1)$ & $21(4.2)$ & $15(3.4)$ & $14(3.0)$ & $21(4.5)$ \\
\hline Other & $12(2.5)$ & $9(1.8)$ & $14(2.8)$ & $11(2.5)$ & $7(1.5)$ & $12(2.6)$ \\
\hline \multicolumn{7}{|c|}{ Baseline $\mathrm{BMI}^{\mathrm{b}}\left[\mathrm{kg} / \mathrm{m}^{2}\right]$} \\
\hline Mean (SD) & $34.8(8.28)$ & $33.9(8.03)$ & $33.5(6.92)$ & $34.5(8.17)$ & $33.5(7.86)$ & $33.6(7.06)$ \\
\hline \multicolumn{7}{|c|}{ Baseline pain intensity score ${ }^{\mathrm{c}, \mathrm{d}}$} \\
\hline Mean (SD) & $7.3(1.28)$ & $7.4(1.28)$ & $7.3(1.20)$ & $7.3(1.28)$ & $7.4(1.27)$ & $7.3(1.22)$ \\
\hline
\end{tabular}

$B M I$ body mass index, $C R$ controlled release, $E R$ extended release, $S D$ standard deviation

${ }^{a}$ Percentages may not total $100 \%$ because of rounding

b History of hypertension: placebo, $n=474$; tapentadol ER, $n=485$; oxycodone CR, $n=495$; listed concomitant antihypertensive medication: placebo, $n=442$; tapentadol ER, $n=458$; oxycodone CR, $n=464$

c History of hypertension: placebo, $n=475$; tapentadol ER, $n=488$; oxycodone CR, $n=499$; listed concomitant antihypertensive medication: placebo, $n=444$; tapentadol ER, $n=461$; oxycodone CR, $n=469$

${ }^{\mathrm{d}}$ Baseline pain intensity was defined as the average of available pain intensity scores (11-point numerical rating scale) during the last $72 \mathrm{~h}$ prior to randomization

between the tapentadol ER and placebo groups or between the tapentadol ER and oxycodone CR groups in either cohort. For patients in both cohorts, a decrease was the most commonly observed change in heart rate from baseline to endpoint and was observed in similar percentages of patients across all treatment groups (Fig. 2). In both patient cohorts, the second most commonly observed change in heart rate from baseline to endpoint across all treatment groups was no change or an increase of $<10 \mathrm{bpm}$ (Fig. 2).

\subsection{Systolic Blood Pressure}

Mean SBP values over time are summarized in Fig. 3. LSM changes from baseline to endpoint in SBP were not significantly different between the tapentadol ER and placebo groups or between the tapentadol ER and oxycodone CR groups in either cohort. Significant differences were observed in the LSM changes from baseline to endpoint in SBP between the oxycodone $\mathrm{CR}$ and placebo groups in patients with at least one listed concomitant antihypertensive medication $(p=0.0442)$, but not in patients with a listed medical history of hypertension ( $p=0.1328)$. LSM changes from baseline to endpoint in SBP were small $(<4 \mathrm{mmHg})$ across all treatment groups in both patient cohorts (Table 2).

The percentages of patients in each of the categories of SBP changes $(<0, \geq 0$ to $<5, \geq 5$ to $<10, \geq 10$ to $<15$, and $\geq 15 \mathrm{mmHg}$ ) from baseline to endpoint are summarized in Fig. 4. There were no significant differences in the overall distribution of categories of SBP changes from baseline to endpoint between the tapentadol ER and placebo groups or between the tapentadol ER and oxycodone CR groups in either patient cohort. The most common change in SBP from baseline to endpoint was a decrease in all treatment groups for patients in both cohorts; similar percentages of patients in the tapentadol ER and oxycodone CR treatment groups and a slightly lower percentage of patients in the placebo group had a decrease in SBP from baseline to endpoint (Fig. 4). The second most commonly observed change in SBP was no change or an increase of $<5 \mathrm{mmHg}$ (Fig. 4). In both patient cohorts, there were no significant differences between the tapentadol ER and 
Fig. 1 Mean heart rate over time for a patients with a listed medical history of hypertension and $\mathbf{b}$ patients with at least one listed concomitant antihypertensive medication (safety population). $B L$ baseline, bpm beats per minute, $C R$ controlled release, $E R$ extended release

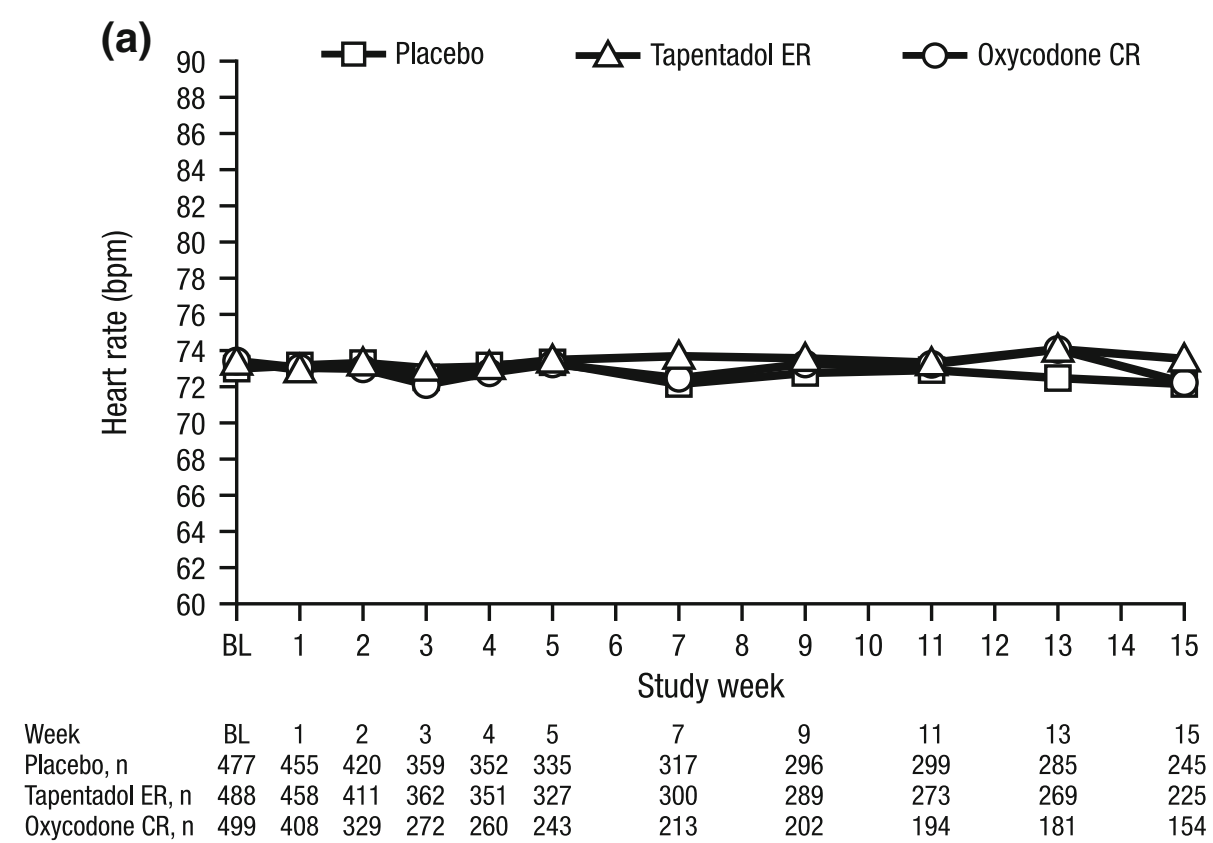

placebo groups in the percentages of patients with sustained (observed over three or more consecutive visits) increases in SBP of $\geq 5, \geq 10$, or $\geq 15 \mathrm{mmHg}$ (Table 3 ).

\subsection{Diastolic Blood Pressure}

Mean DBP values over time are summarized in Fig. 5. For patients in both cohorts, LSM changes from baseline to endpoint in DBP were small $(<3 \mathrm{mmHg})$ across all treatment groups (Table 2), and no significant differences were observed in LSM changes from baseline to endpoint in DBP between the tapentadol ER and placebo groups or between the tapentadol ER and oxycodone CR groups. The differences in the LSM changes from baseline to endpoint in DBP between the oxycodone $\mathrm{CR}$ and placebo groups were significant in patients with a listed medical history of hypertension $(p=0.0159)$ and patients with at least one listed concomitant antihypertensive medication $(p=0.0032)$.

The percentages of patients in each category of DBP change $(<0, \geq 0$ to $<5, \geq 5$ to $<10, \geq 10$ to $<15$, and 
Table 2 Least squares mean (standard error) changes from baseline to endpoint in heart rate and blood pressure for patients with a listed medical history of hypertension and for patients with at least one listed concomitant antihypertensive medication (safety population)

\begin{tabular}{llllllll}
\hline Vital sign & \multicolumn{2}{l}{ Listed medical history of hypertension } & & \multicolumn{2}{l}{ Listed concomitant antihypertensive medication } \\
\cline { 2 - 3 } & $\begin{array}{l}\text { Placebo } \\
(n=459)\end{array}$ & $\begin{array}{l}\text { Tapentadol ER } \\
(n=462)\end{array}$ & $\begin{array}{l}\text { Oxycodone CR } \\
(n=413)\end{array}$ & $\begin{array}{l}\text { Placebo } \\
(n=429)\end{array}$ & $\begin{array}{l}\text { Tapentadol ER } \\
(n=434)\end{array}$ & $\begin{array}{l}\text { Oxycodone CR } \\
(n=387)\end{array}$ \\
\hline Heart rate (bpm) & $-0.7(0.44)$ & $0.2(0.43)$ & $-0.9(0.45)$ & & $-0.6(0.45)$ & $0.1(0.44)$ & $-0.7(0.47)$ \\
SBP (mmHg) & $-2.4(0.64)$ & $-2.7(0.64)$ & $-3.7(0.67)$ & & $-1.8(0.66)$ & $-3.3(0.65)$ & $-3.7(0.69)^{*}$ \\
DBP (mmHg) & $-1.0(0.39)$ & $-1.3(0.39)$ & $-2.3(0.41)^{* *}$ & & $-0.7(0.40)$ & $-1.4(0.40)$ & $-2.3(0.42)^{* * *}$ \\
\hline
\end{tabular}

bpm beats per minute, $C R$ controlled release, $D B P$ diastolic blood pressure, $E R$ extended release, $S B P$ systolic blood pressure $* p=0.0442, * * p=0.0159, * * * p=0.0032$ versus placebo

Fig. 2 Categories of heart rate changes from baseline at endpoint for patients with a listed medical history of hypertension and for patients with at least one listed concomitant antihypertensive medication (safety population). bpm beats per minute, $C R$ controlled release, $E R$ extended release
Decrease

$\square$ Increase of 15 to $<20 \mathrm{bpm}$
Increase of 10 to $<15 \mathrm{bpm}$

$\mathbf{N}$ Increase of $20 \mathrm{bpm}$

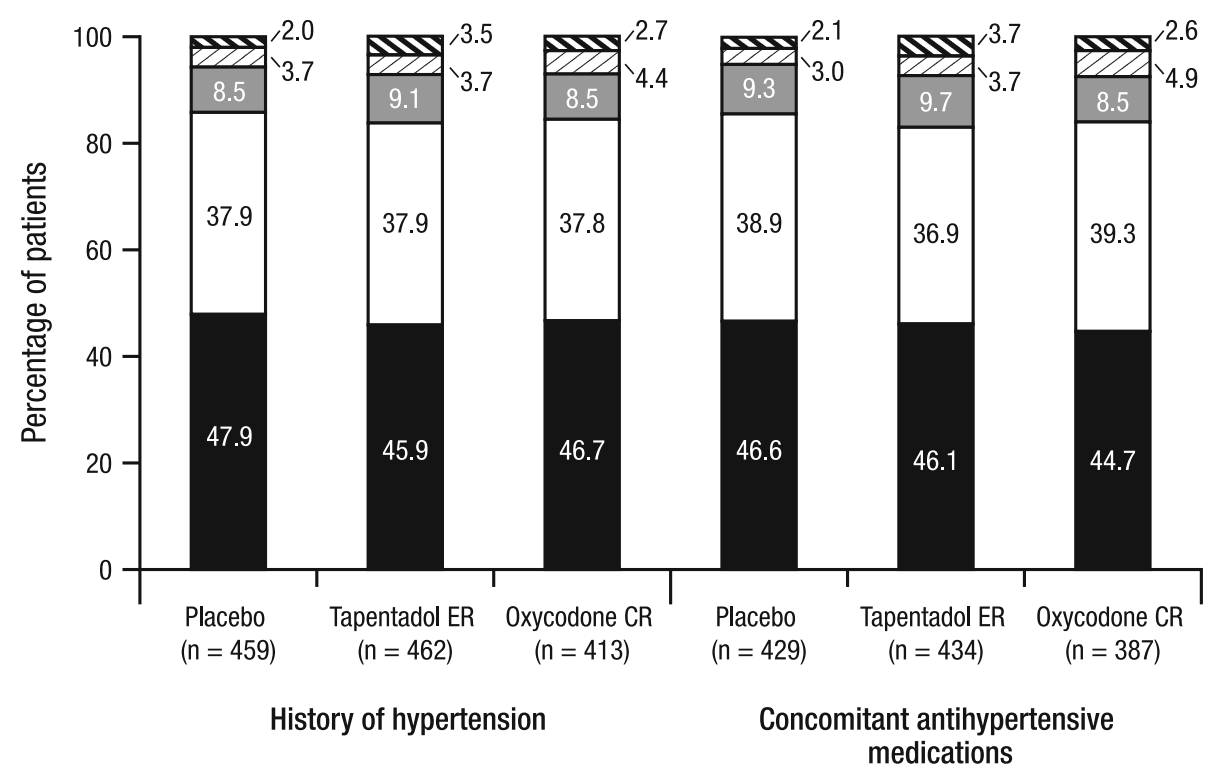

$\geq 15 \mathrm{mmHg}$ ) from baseline to endpoint are summarized in Fig. 6. There were no significant differences between the tapentadol ER and placebo groups or between the tapentadol ER and oxycodone CR groups in the overall distribution of categories of DBP changes from baseline to endpoint in either patient cohort. Across all treatment groups, the most commonly observed change in DBP from baseline to endpoint was a decrease in DBP in both patient cohorts; the percentage of patients with a decrease in DBP from baseline to endpoint was comparable in the placebo and tapentadol ER groups and slightly higher in the oxycodone CR group in both cohorts (Fig. 6). The second most commonly observed change in DBP was in the category of no change or an increase of $\geq 0$ to $<5 \mathrm{mmHg}$ (Fig. 6). In both cohorts, there were no significant differences between the tapentadol ER and placebo groups in the percentages of patients with sustained (observed over three or more consecutive visits) increases in DBP of $\geq 5$, $\geq 10$, or $\geq 15 \mathrm{mmHg}$ (Table 3); however, in both cohorts, a significantly lower percentage of patients in the oxycodone CR group than in the tapentadol ER group had sustained increases in DBP of $\geq 5 \mathrm{mmHg}$ ( $p<0.001$ for both comparisons), but between-group differences in the percentages of patients with sustained increases of $\geq 10$ or $\geq 15 \mathrm{mmHg}$ were not significant (Table 3 ). Less than $4 \%$ of patients in any treatment group in both patient cohorts had sustained increases from baseline in DBP of $\geq 15 \mathrm{mmHg}$ (Table 3).

\subsection{Cardiovascular Treatment-Emergent Adverse Events}

In patients with a listed medical history of hypertension, cardiovascular TEAEs were reported in the placebo, tapentadol ER, and oxycodone CR groups for $6.1 \%$ 
Fig. 3 Mean systolic blood pressure over time for a patients with a listed medical history of hypertension and $\mathbf{b}$ patients with at least one listed concomitant antihypertensive medication (safety population). $B L$ baseline, $C R$ controlled release, $E R$ extended release, SBP systolic blood pressure
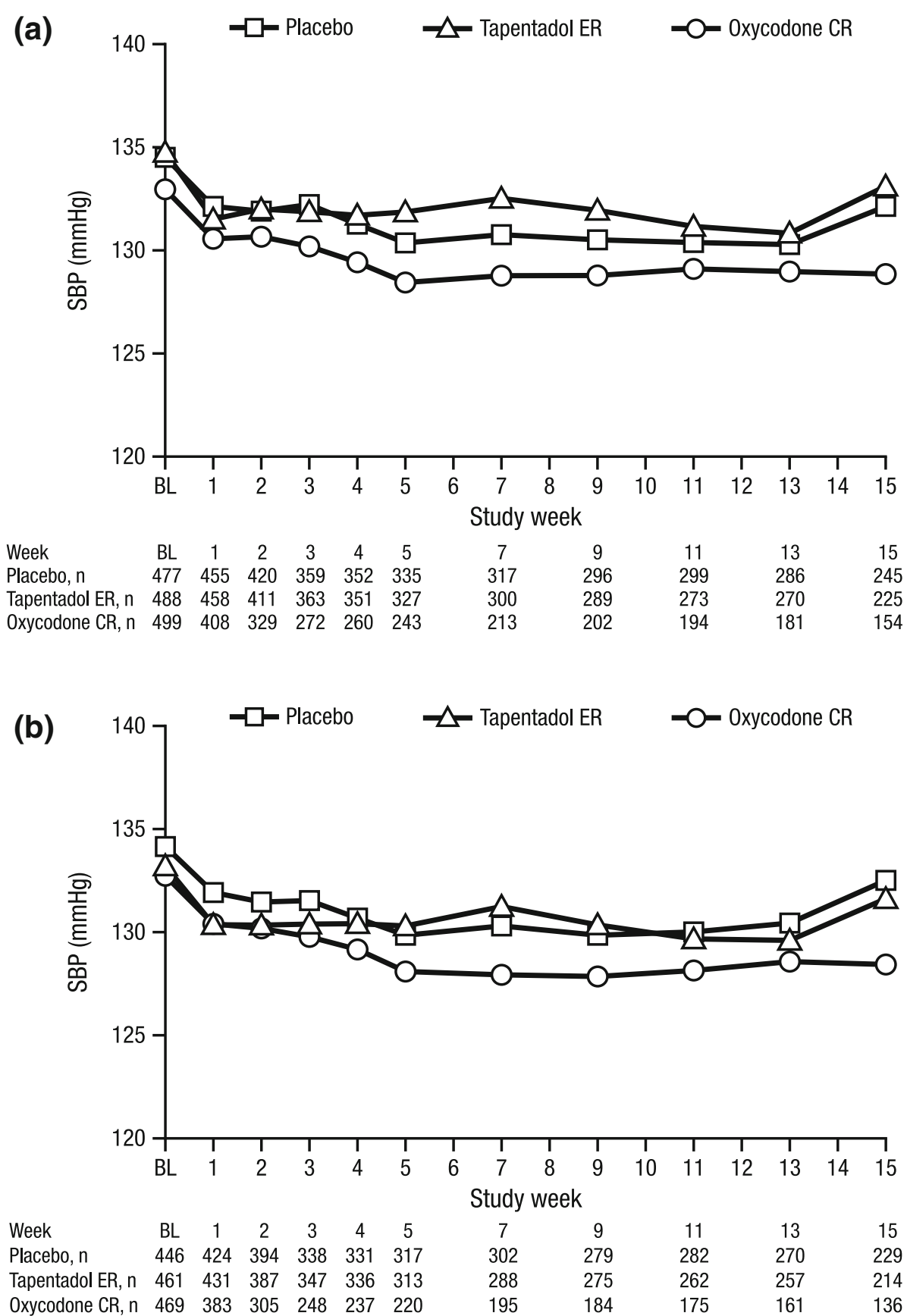

(29/477), $8.6 \%$ (42/488), and $11.2 \%$ (56/499) of patients, respectively. In patients with at least one listed concomitant antihypertensive medication, cardiovascular TEAEs were reported in the placebo, tapentadol ER, and oxycodone CR groups for $5.2 \%$ (23/446), $8.5 \%$ (39/461), and $11.9 \%(56 / 469)$ of patients, respectively. The most common cardiovascular TEAEs (incidence $\geq 1 \%$ in any treatment group in either cohort) are summarized in Table 4. A total of seven patients experienced serious cardiovascular TEAEs; all seven patients were included in both patient cohorts. One patient in the placebo group experienced atrial fibrillation (considered serious and unlikely to be related to study treatment). One patient in the tapentadol ER group experienced atrial fibrillation (considered serious and possibly related to study treatment). Five patients in the oxycodone CR group experienced serious cardiovascular TEAEs (considered not related or unlikely to be related to study treatment); two of these patients experienced myocardial infarction, one experienced angina pectoris and coronary artery occlusion, one experienced palpitations, and one experienced angina pectoris. 
Fig. 4 Categories of systolic blood pressure changes from baseline at endpoint for patients with a listed medical history of hypertension and for patients with at least one listed concomitant antihypertensive medication (safety population). $C R$ controlled release, $E R$ extended release
Decrease

Increase of 0 to $<5 \mathrm{mmHg}$

Increase of 5 to $<10 \mathrm{mmHg}$

$\square$ Increase of 10 to $<15 \mathrm{mmHg} \quad \mathbf{N}$ Increase of $15 \mathrm{mmHg}$

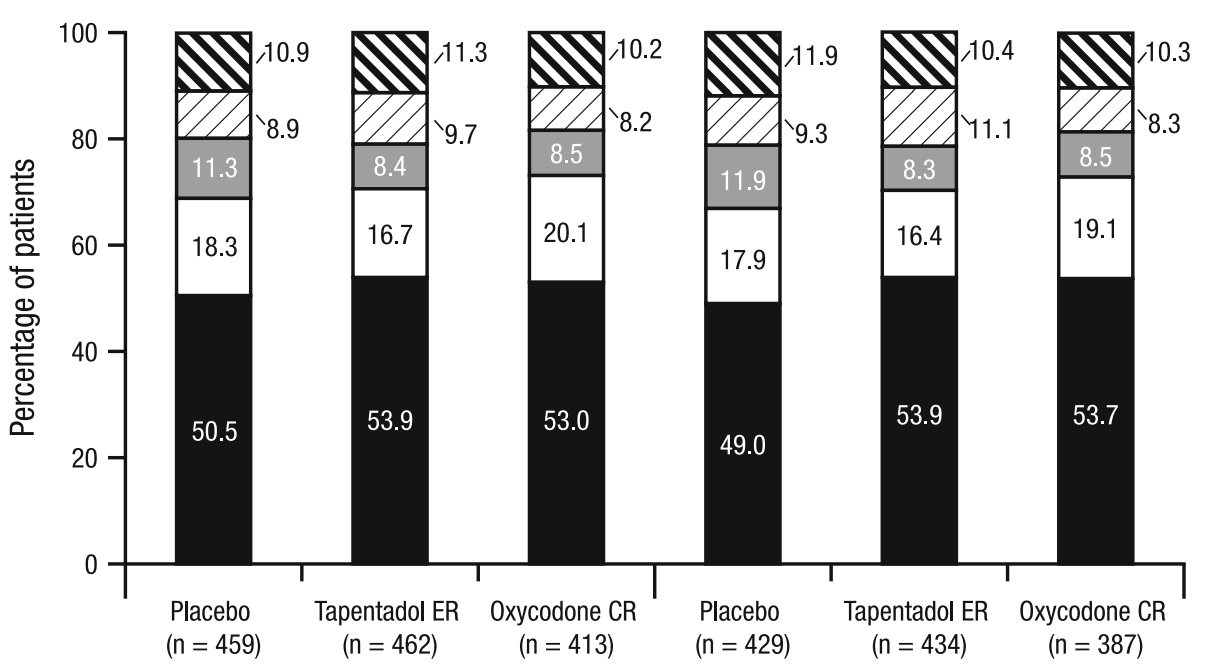

History of hypertension
Concomitant antihypertensive medications

Table 3 Numbers and percentages of patients in each category of sustained systolic or diastolic blood pressure increase in the cohort of patients with a listed medical history of hypertension and in the cohort of patients with at least one listed concomitant antihypertensive medication (safety population) ${ }^{\mathrm{a}}$

\begin{tabular}{|c|c|c|c|c|c|c|}
\hline \multirow{2}{*}{$\begin{array}{l}\text { Sustained increase } \\
\text { category }[n(\%)]\end{array}$} & \multicolumn{3}{|c|}{ Listed medical history of hypertension } & \multicolumn{3}{|c|}{ Listed concomitant antihypertensive medication } \\
\hline & $\begin{array}{l}\text { Placebo } \\
(n=459)\end{array}$ & $\begin{array}{l}\text { Tapentadol ER } \\
(n=462)\end{array}$ & $\begin{array}{l}\text { Oxycodone CR } \\
(n=413)\end{array}$ & $\begin{array}{l}\text { Placebo } \\
(n=429)\end{array}$ & $\begin{array}{l}\text { Tapentadol ER } \\
(n=434)\end{array}$ & $\begin{array}{l}\text { Oxycodone CR } \\
(n=387)\end{array}$ \\
\hline \multicolumn{7}{|l|}{ SBP } \\
\hline$\geq 5 \mathrm{mmHg}$ & $130(28.3)$ & 109 (23.6) & $69(16.7)^{*}$ & $121(28.2)$ & $105(24.2)$ & $64(16.5)^{* *}$ \\
\hline$\geq 10 \mathrm{mmHg}$ & $72(15.7)$ & $69(14.9)$ & $41(9.9)^{*}$ & $69(16.1)$ & $65(15.0)$ & $37(9.6)^{*}$ \\
\hline$\geq 15 \mathrm{mmHg}$ & $32(7.0)$ & $29(6.3)$ & $17(4.1)$ & $30(7.0)$ & $28(6.5)$ & $16(4.1)$ \\
\hline \multicolumn{7}{|l|}{ DBP } \\
\hline$\geq 5 \mathrm{mmHg}$ & $117(25.5)$ & $100(21.6)$ & $53(12.8) * * *$ & $113(26.3)$ & $96(22.1)$ & $48(12.4)^{* * *}$ \\
\hline$\geq 10 \mathrm{mmHg}$ & $56(12.2)$ & $45(9.7)$ & $33(8.0)$ & $50(11.7)$ & $44(10.1)$ & $26(6.7)$ \\
\hline$\geq 15 \mathrm{mmHg}$ & $17(3.7)$ & $15(3.2)$ & $7(1.7)$ & $13(3.0)$ & $15(3.5)$ & $7(1.8)$ \\
\hline
\end{tabular}

$C R$ controlled release, $D B P$ diastolic blood pressure, $E R$ extended release, SBP systolic blood pressure

$* p<0.05, * * p<0.01, * * * p<0.001$ versus tapentadol ER

a A sustained increase was defined as an increase observed over $\geq 3$ consecutive visits

\section{Discussion}

This post hoc pooled analysis of data from three randomized, placebo- and active-controlled, phase III studies [18, 20] evaluated blood pressure and heart rate in patients with a listed medical history of hypertension or at least one listed concomitant antihypertensive medication at baseline who were treated with tapentadol ER (100-250 mg twice daily) for the management of chronic pain. Mean heart rate, SBP, and DBP values remained generally unchanged over 15 weeks of treatment with placebo, tapentadol ER
(100-250 mg twice daily), or oxycodone $\mathrm{HCl}$ CR (20-50 mg twice daily) in both patient cohorts. LSM changes from baseline to endpoint in heart rate, SBP, and DBP in all treatment groups were generally small and negative for all three parameters in both patient cohorts. No significant differences were observed between tapentadol ER and placebo for any of the evaluated parameters. Based on the categorical analyses of changes in heart rate, SBP, and DBP, increases in these three parameters were observed for approximately $45-55 \%$ of patients in all three treatment groups; however, results of these analyses 
Fig. 5 Mean diastolic blood pressure over time for a patients with a listed medical history of hypertension and $\mathbf{b}$ patients with at least one listed concomitant antihypertensive medication (safety population). $B L$ baseline, $C R$ controlled release, $D B P$ diastolic blood pressure, $E R$ extended release

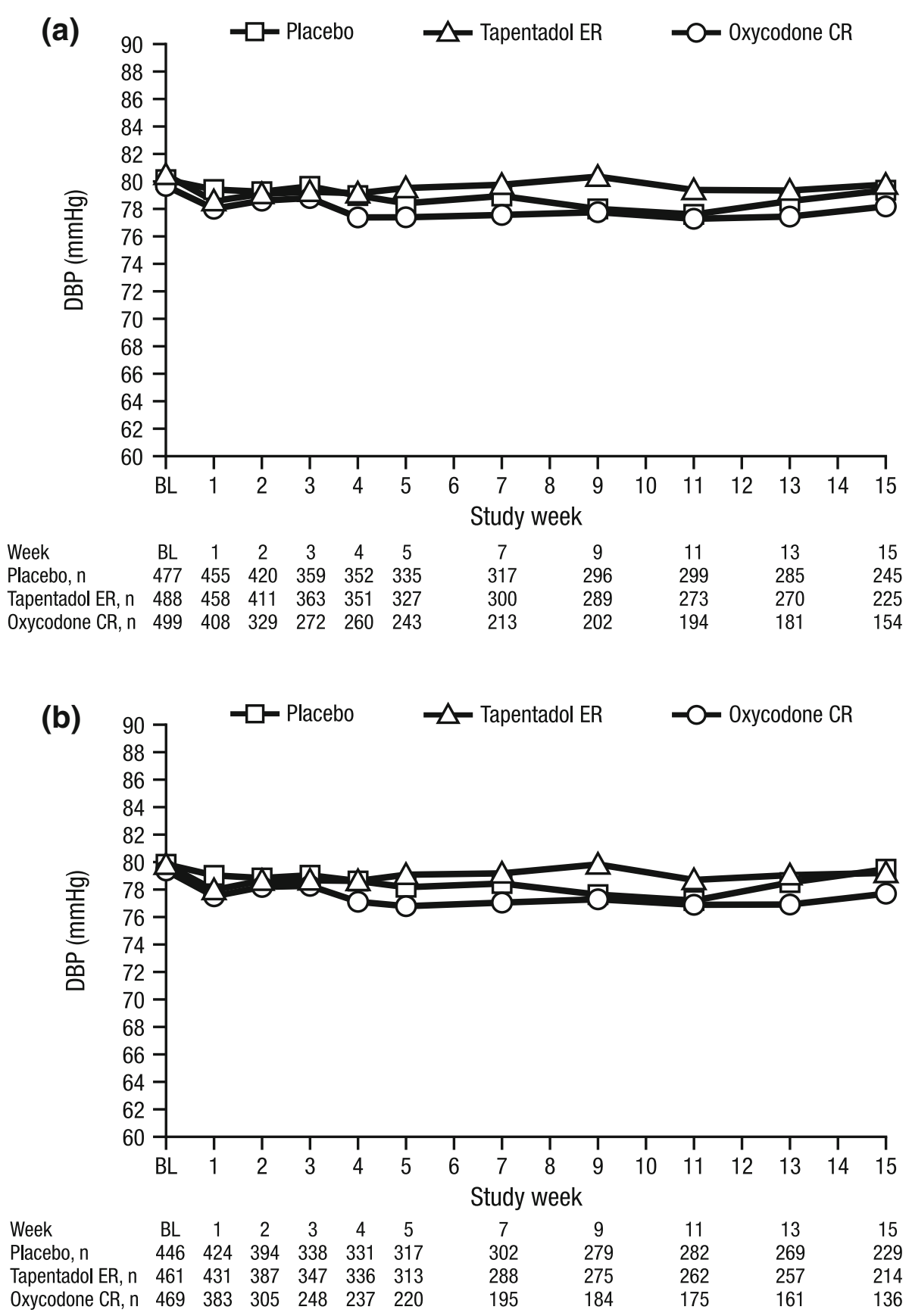

were generally comparable for the tapentadol ER and placebo treatment groups. In patients with a listed history of hypertension and in patients with at least one listed concomitant antihypertensive medication, respectively, decreases of 2.7 and $3.3 \mathrm{mmHg}$ for SBP and 1.3 and $1.4 \mathrm{mmHg}$ for DBP were observed after 15 weeks of treatment with tapentadol ER (based on LSM changes from baseline to endpoint).

The most commonly reported change in heart rate and blood pressure in all treatment groups across both cohorts was a decrease in heart rate or blood pressure, and only a few patients experience a sustained (observed over three or more consecutive visits) increase in SBP or DBP. Generally, decreases in DBP were observed for a higher percentage of patients in the oxycodone CR group than in the placebo or tapentadol ER groups across both cohorts. $\mu$-opioid receptor agonists have been associated with decreases in blood pressure [24]; oxycodone is a pure opioid agonist, while tapentadol also has norepinephrine reuptake inhibitor activity, which may account for the observed differences. The incidence of cardiovascular TEAEs overall and the incidence of serious cardiovascular 
Fig. 6 Categories of diastolic blood pressure changes from baseline at endpoint for patients with a listed medical history of hypertension and for patients with at least one listed concomitant antihypertensive medication (safety population). $C R$ controlled release, $E R$ extended release
Increase of 10 to $<15 \mathrm{mmHg} \quad \mathbf{V}$ Increase of $15 \mathrm{mmHg}$

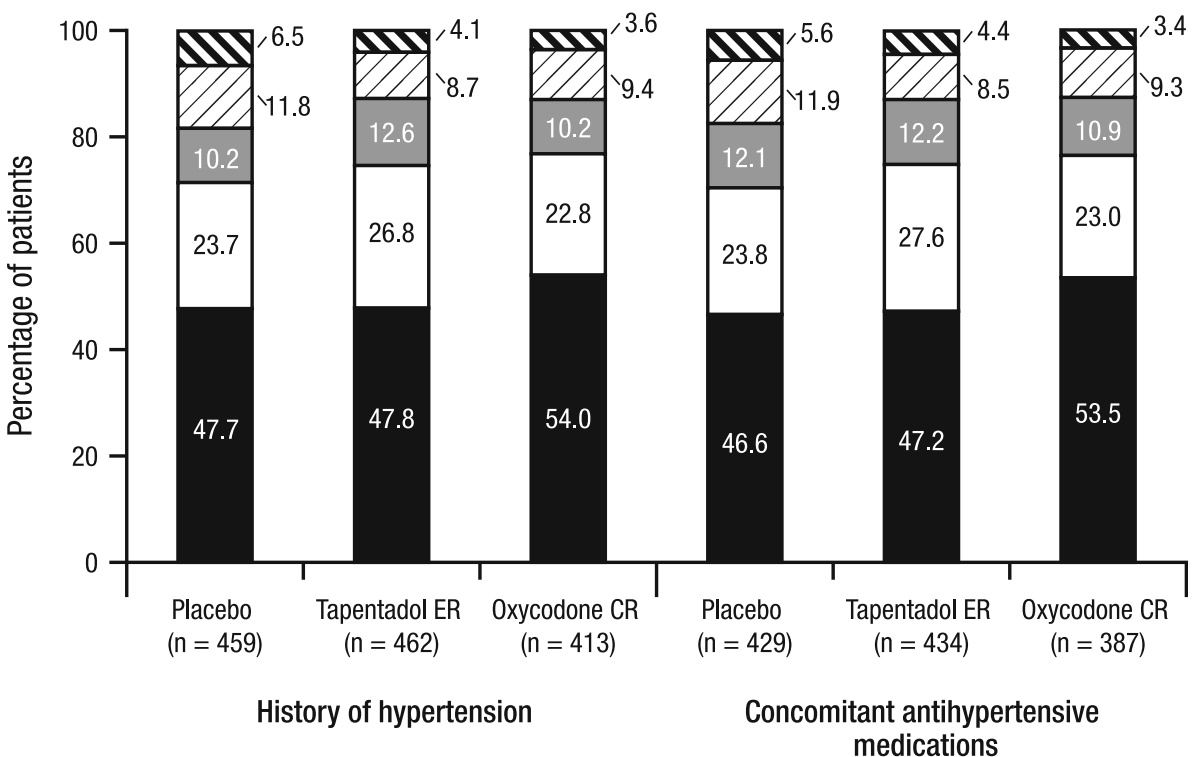

Table 4 Cardiovascular treatment-emergent adverse events reported for $\geq 1 \%$ of patients in any treatment group in the cohort of patients with a listed medical history of hypertension and in the cohort of patients with at least one listed concomitant antihypertensive medication (safety population)

\begin{tabular}{|c|c|c|c|c|c|c|}
\hline \multirow{2}{*}{$\begin{array}{l}\text { System organ class } \\
\text { Preferred term }[n(\%)]\end{array}$} & \multicolumn{3}{|c|}{ Listed medical history of hypertension } & \multicolumn{3}{|c|}{ Listed concomitant antihypertensive medication } \\
\hline & $\begin{array}{l}\text { Placebo } \\
(n=477)\end{array}$ & $\begin{array}{l}\text { Tapentadol ER } \\
(n=489)\end{array}$ & $\begin{array}{l}\text { Oxycodone CR } \\
(n=499)\end{array}$ & $\begin{array}{l}\text { Placebo } \\
(n=446)\end{array}$ & $\begin{array}{l}\text { Tapentadol ER } \\
(n=461)\end{array}$ & $\begin{array}{l}\text { Oxycodone CR } \\
(n=469)\end{array}$ \\
\hline Any cardiovascular TEAE & $29(6.1)$ & $42(8.6)$ & $56(11.2)$ & $23(5.2)$ & $39(8.5)$ & $56(11.9)$ \\
\hline Cardiac disorders & $16(3.4)$ & $18(3.7)$ & $32(6.4)$ & $11(2.5)$ & $14(3.0)$ & $33(7.0)$ \\
\hline Palpitations & $1(0.2)$ & $2(0.4)$ & $8(1.6)$ & $1(0.2)$ & $2(0.4)$ & $10(2.1)$ \\
\hline Angina pectoris & $1(0.2)$ & 0 & $5(1.0)$ & $1(0.2)$ & 0 & $6(1.3)$ \\
\hline Vascular disorders & $16(3.4)$ & $26(5.3)$ & $30(6.0)$ & $14(3.1)$ & $26(5.6)$ & $29(6.2)$ \\
\hline Hypertension & $12(2.5)$ & $12(2.5)$ & $11(2.2)$ & $11(2.5)$ & $12(2.6)$ & $11(2.3)$ \\
\hline Hot flush & $1(0.2)$ & $9(1.8)$ & $10(2.0)$ & $1(0.2)$ & $9(2.0)$ & $9(1.9)$ \\
\hline Flushing & $1(0.2)$ & $1(0.2)$ & $4(0.8)$ & $1(0.2)$ & $1(0.2)$ & $5(1.1)$ \\
\hline
\end{tabular}

$C R$ controlled release, $E R$ extended release, TEAE treatment-emergent adverse event

TEAEs in the tapentadol ER group was low and similar to incidences observed in the placebo group. These results are consistent with previous observations from premarketing analyses of data from ten phase II or III studies of tapentadol ER showing that cardiac adverse drug reactions (specifically heart rate increased, palpitations, heart rate decreased, and left bundle branch block) were reported in less than $1 \%$ of patients taking tapentadol ER, while vascular adverse drug reactions were reported in no more than $2 \%$ of patients (blood pressure decreased, $<1 \%$; hot flush, $2 \%$ ) [17].

Acetaminophen, NSAIDs, and COX-2 inhibitors are generally considered to be the foundation of early treatment options for chronic osteoarthritis pain [3-5] and low back pain $[3,6,7]$. The use of these analgesics in patients with a current or prior history of cardiovascular disease, including hypertension, has been associated with increases in blood pressure [8] (a risk factor for cardiovascular events) and an increased risk for cardiovascular events [4-6, 25, 26]. Significant increases in mean SBP (of up to $14.3 \mathrm{mmHg}$ ) and DBP (of up to $2.3 \mathrm{mmHg}$ ) have also been observed with NSAIDs and COX-2 inhibitors in patients with treated hypertension [9]. In the current study, no significant increases in the LSM changes in SBP or DBP were observed in the cohort of patients with a listed medical history of hypertension or the cohort of patients receiving at least one listed concomitant antihypertensive medication in the tapentadol ER or oxycodone CR groups. 
The retrospective post hoc design of this analysis and use of data from patients involved in clinical trials may represent limitations for the interpretation and translation of these results to clinical practice. In addition, the permitted use of acetaminophen, which has previously been associated with an increase in blood pressure [8], as rescue medication in the studies included in this pooled analysis may have had an impact on these results; however, on average, the number of days that patients received acetaminophen was low and similar across treatment groups, and the percentage of patients receiving acetaminophen as rescue medication was comparable across treatment groups. Therefore, it is unlikely that the use of acetaminophen would have had a clinically relevant impact on blood pressure measures for this pooled patient population or on blood pressure comparisons between treatment groups. Furthermore, the use of $\beta$-blockers ( $\beta$-adrenoceptor antagonists) as antihypertensive therapy may have had an impact on results; however, the percentage of patients using $\beta$-blockers at baseline was comparable across treatment groups in the overall pooled patient population from these three studies (placebo, $19.6 \%$ [195/993]; tapentadol ER, $20.6 \%$ [202/981]; oxycodone CR, $19.0 \%$ [190/1,001]). Therefore, it is unlikely that $\beta$-blocker use had an effect on comparisons of blood pressure or heart rate between treatment groups.

Results of the current post hoc analysis add to the body of evidence associated with the cardiovascular safety findings for tapentadol. These results are consistent with those from a previous analysis of data pooled from the same three studies, which showed no clinically meaningful changes in mean vital sign measures, including heart rate and blood pressure, in patients with chronic pain who were receiving tapentadol ER (100-250 mg twice daily) treatment [23]. Results of categorical analyses, which showed increases in heart rate and blood pressure for some patients, were generally similar for tapentadol ER and placebo. Findings suggest that tapentadol ER is a reasonable option when considering treatments for the management of chronic pain in patients with hypertension, although a personalized assessment of patient risk and prudent clinical judgment are recommended.

Acknowledgments These analyses were funded by Janssen Scientific Affairs, LLC, and were designed and performed by employees of Janssen Scientific Affairs, LLC. Editorial support for the writing of this manuscript was provided by Megan Knagge, $\mathrm{PhD}$, of MedErgy, and was funded by Janssen Scientific Affairs, LLC. The authors retained full editorial control over the content of the manuscript. David Biondi is an employee of Janssen Scientific Affairs, LLC; Bruce Moskovitz was an employee of Janssen Scientific Affairs, LLC at the time this analysis was performed; and Jim Xiang and Mila Etropolski are employees of Janssen Research \& Development, LLC. All authors are Johnson \& Johnson shareholders.
Open Access This article is distributed under the terms of the Creative Commons Attribution Noncommercial License which permits any noncommercial use, distribution, and reproduction in any medium, provided the original author(s) and the source are credited.

\section{References}

1. Caporali R, Cimmino MA, Sarzi-Puttini P, et al. Comorbid conditions in the AMICA study patients: effects on the quality of life and drug prescriptions by general practitioners and specialists. Semin Arthritis Rheum. 2005;35:31-7.

2. van den Bussche H, Koller D, Kolonko T, et al. Which chronic diseases and disease combinations are specific to multimorbidity in the elderly? Results of a claims data based cross-sectional study in Germany. BMC Public Health. 2011;11:101.

3. Schnitzer TJ. Update on guidelines for the treatment of chronic musculoskeletal pain. Clin Rheumatol. 2006;25(Suppl 7):22-9.

4. Zhang W, Moskowitz RW, Nuki G, et al. OARSI recommendations for the management of hip and knee osteoarthritis, part II: OARSI evidence-based, expert consensus guidelines. Osteoarthr Cartil. 2008;16:137-62.

5. Zhang W, Nuki G, Moskowitz RW, et al. OARSI recommendations for the management of hip and knee osteoarthritis: part III: changes in evidence following systematic cumulative update of research published through January 2009. Osteoarthr Cartil. 2010;18:476-99.

6. Chou R, Qaseem A, Snow V, et al. Diagnosis and treatment of low back pain: a joint clinical practice guideline from the American College of Physicians and the American Pain Society. Ann Intern Med. 2007;147:478-91.

7. Chou R, Huffman LH. Medications for acute and chronic low back pain: a review of the evidence for an American Pain Society/American College of Physicians clinical practice guideline. Ann Intern Med. 2007;147:505-14.

8. Sudano I, Flammer AJ, Periat D, et al. Acetaminophen increases blood pressure in patients with coronary artery disease. Circulation. 2010;122:1789-96.

9. Snowden S, Nelson R. The effects of nonsteroidal anti-inflammatory drugs on blood pressure in hypertensive patients. Cardiol Rev. 2011;19:184-91.

10. Zhang J, Ding EL, Song Y. Adverse effects of cyclooxygenase 2 inhibitors on renal and arrhythmia events: meta-analysis of randomized trials. JAMA. 2006;296:1619-32.

11. CYMBALTA $^{\circledR}$ (duloxetine hydrochloride) delayed-release capsules [package insert]. Indianapolis: Eli Lilly and Company; 2011.

12. Stahl SM, Grady MM, Moret C, et al. SNRIs: their pharmacology, clinical efficacy, and tolerability in comparison with other classes of antidepressants. CNS Spectr. 2005;10:732-47.

13. Mayer AF, Schroeder C, Heusser K, et al. Influences of norepinephrine transporter function on the distribution of sympathetic activity in humans. Hypertension. 2006;48:120-6.

14. Tzschentke TM, Christoph T, Kögel B, et al. (-)-(1R,2R)-3-(3dimethylamino-1-ethyl-2-methyl-propyl)-phenol hydrochloride (tapentadol $\mathrm{HCl}$ ): a novel m-opioid receptor agonist/norepinephrine reuptake inhibitor with broad-spectrum analgesic properties. J Pharmacol Exp Ther. 2007;323:265-76.

15. Tzschentke TM, De Vry J, Terlinden R, et al. Tapentadol hydrochloride. Analgesic, mu-opioid receptor agonist, noradrenaline reuptake inhibitor. Drugs Future. 2006;31:1053-61.

16. Tzschentke TM, Jahnel U, Kogel B, et al. Tapentadol hydrochloride: a next-generation, centrally acting analgesic with two 
mechanisms of action in a single molecule. Drugs Today (Barc). 2009;45:483-96.

17. Nucynta ${ }^{\circledR}$ ER (tapentadol) extended-release oral tablets C-II [package insert]. Raritan: Janssen Pharmaceuticals, Inc.; 2011.

18. Afilalo M, Etropolski MS, Kuperwasser B, et al. Efficacy and safety of tapentadol extended release compared with oxycodone controlled release for the management of moderate to severe chronic pain related to osteoarthritis of the knee: a randomized, double-blind, placebo- and active-controlled phase III study. Clin Drug Investig. 2010;30:489-505.

19. Wild JE, Grond S, Kuperwasser B, et al. Long-term safety and tolerability of tapentadol extended release for the management of chronic low back pain or osteoarthritis pain. Pain Pract. 2010;10:416-27.

20. Buynak R, Shapiro DY, Okamoto A, et al. Efficacy and safety of tapentadol extended release for the management of chronic low back pain: results of a prospective, randomized, double-blind, placebo- and active-controlled phase III study. Expert Opin Pharmacother. 2010;11:1787-804.

21. Schwartz S, Etropolski M, Shapiro DY, et al. Safety and efficacy of tapentadol ER in patients with painful diabetic peripheral neuropathy: results of a randomized-withdrawal, placebo-controlled trial. Curr Med Res Opin. 2011;27:151-62.

22. Lange B, Kuperwasser B, Okamoto A, et al. Efficacy and safety of tapentadol prolonged release for chronic osteoarthritis pain and low back pain. Adv Ther. 2010;27:381-99.

23. Etropolski M, Shapiro D, Okamoto A, et al. An analysis of changes in vital signs and electrocardiogram measurements with tapentadol prolonged release (PR) treatment [abstract no. F644]. 7 th Congress of the European Federation of IASP Chapters; 21-24 Sep 2011; Hamburg.

24. Czapla MA, Gozal D, Alea OA, et al. Differential cardiorespiratory effects of endomorphin 1, endomorphin 2, DAMGO, and morphine. Am J Respir Crit Care Med. 2000;162:994-9.

25. Kearney PM, Baigent C, Godwin J, et al. Do selective cyclooxygenase-2 inhibitors and traditional non-steroidal anti-inflammatory drugs increase the risk of atherothrombosis? Meta-analysis of randomised trials. BMJ. 2006;332:1302-8.

26. Pham K, Hirschberg R. Global safety of coxibs and NSAIDs. Curr Top Med Chem. 2005;5:465-73. 